\title{
VISIBLE/UV SCATTERING BY INTERSTELLAR DUST
}

\author{
A. N. WITT \\ The University of Toledo
}

\begin{abstract}
Studies of interstellar dust based on observations of scattered and dust-emitted radiation from grains are reviewed.
\end{abstract}

\section{INTRODUCTION}

Methods for the observational study of interstellar dust can be divided into direct and indirect approaches. While the latter depend on the observation of background sources of all kinds and make inferences about the dust based on detected changes in e. g. energy distributions, polarization states, or number counts, the direct method depends on the analysis of radiation emanating from the dust itself, either through scattering or emission. Here I will review a number of topics which have emerged from the direct study of interstellar dust in the UV/visible spectral region, roughly defined as the wavelength range from the Lyman limit to $1.0 \mu \mathrm{m}$.

Scattering by interstellar dust is an important process, as was recognized early by Slipher (1912) with the detection of continuous spectra from reflection nebulae. Hubble (1922) derived a first quantitative estimate for the relatively high value of the dust albedo in the visible from an observed relationship between the apparent radii of reflection nebulae and the magnitudes of their respective illuminating stars. The presence of the diffuse galactic light, detected first by Elvey and Roach (1937) and subsequently investigated by Henyey and Greenstein (1941), demonstrated that similar high-albedo dust was abundant throughout the diffuse interstellar medium. As one views the Milky Way, approximately $25 \%$ of the total integrated light is due to the diffusely scattered component.

\section{CHARACTERIZATION OF SCATTERING PROPERTIES}

\section{1. $A L B E D O$}

We define the albedo $a$ for single scattering as the ratio of the total scattering cross section to the total extinction cross section. When given for a single grain, the albedo is a function of grain size, composition, and wavelength $\lambda$. When measured in an interstellar system, the albedo is always averaged over the actual distribution of sizes and compositions of grains responsible for the extinction over the same pathlength. If Mie theory is taken as a guide, there is only limited information concerning composition, somewhat more information concerning particle size, to be obtained from an albedo measurement, as is shown in Table $1 . \quad$ Useful 
TABLE 1

Albedo Values Predicted from MIE Theory

\begin{tabular}{ccc}
\hline \hline Grain Size & Dielectric Material & Absorbing Material \\
\hline & & $\rightarrow 0$ \\
$<<\lambda$ & 1 & $\sim 0.3-0.8$ \\
$\sim \lambda$ & 1 & 0.5 \\
$>>\lambda$ & 1 & \\
\hline
\end{tabular}

information can come from results on the wavelength dependence of the albedo over a wavelength range sufficiently wide, so that the dominance of different grain populations in different spectral regions can be detected. Of special interest is the variation of the albedo with wavelength across interstellar extinction features, such as the $2175 \AA$ band in the UV, or the diffuse interstellar features in the visible.

\subsection{PHASE FUNCTION}

The angular redistribution of the light scattered by a dust grain is described by the phase function, $\phi(\cos \alpha)$, where $\alpha$ is the scattering angle. The general shape of the phase function varies from a nearly isotropic form for grains small compared to the wavelength to a strongly forward directed shape for grains of sizes comparable to or larger than the wavelength. This variation in shape is measured by the asymmetry parameter, $g$, defined as:

$$
g=1 / 2 \int_{-1}^{1} \phi(\cos \alpha) \cdot \cos \alpha \cdot d(\cos \alpha)=\langle\cos \alpha\rangle
$$

The range of values of interest for interstellar dust extends from $g=0$ (isotropic) to $g=+1$ (total forward-scattering). As the ratio of grain diameter to wavelength changes from 0.3 to 1.0 , a range of particular interest for interstellar dust, the value of $g$ undergoes a drastic change from about +0.15 to +0.75 . Hence, the phase function asymmetry parameter is a sensitive function of grain size, and its determination as a function of wavelength can give useful information on the nature of the grain size distribution.

\subsection{THE HENYEY-GREENSTEIN PHASE FUNCTION}

The derivation of dust scattering properties usually involves the comparison of diffuse light measurements with predictions from radiative transfer models. The question of what to use for the phase function in model calculations is frequently resolved with the choice of the single-parameter Henyey-Greenstein (1941) phase function $\phi_{H G}$ :

$$
\phi_{H G}(\cos \alpha)=\frac{1}{4 \pi}\left(1-g^{2}\right)\left(1+g^{2}-2 g \cos \alpha\right)^{-\frac{3}{2}}
$$

In Figure 1, phase functions for two spherical, homogeneous enstatite grains computed from Mie theory are compared with HG phase functions with identical asymmetry parameters. 


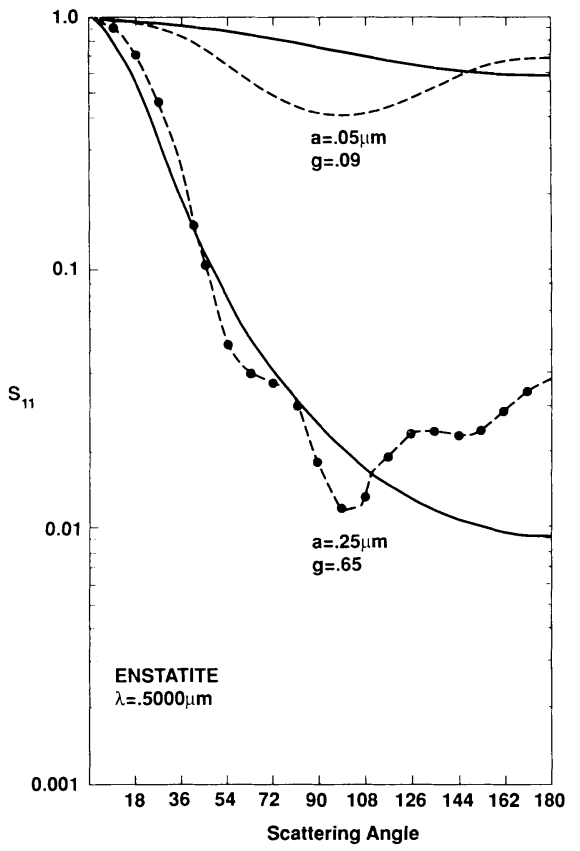

Fig. 1. Comparison of the phase function of two spherical enstatite grains computed from Mie theory (dashed curves) with Henyey-Greenstein phase functions (solid curves) with identical asymmetry parameters.

As the comparison shows, the HG phase function is not a good representation for the Rayleigh-like scattering pattern of small grains (e.g. radius $a=0.05 \mu \mathrm{m}$ ) at intermediate scattering angles, nor does it represent the back-scattering peak of larger spheres (e.g. radius $a=0.25 \mu \mathrm{m}$ ) well at all. On the other hand, it provides a fair match for the pronounced forward-scattering peak, where most of the light scattered by larger grains is found. Certainly, in any model situation dominated by forward-scattering, the HG phase function is an appropriate choice.

Another situation often encountered is one where scattering occurs over a large range of scattering angles. Under such conditions, a measure for the performance of a phase function is $f$, the fraction of light scattered into the forward hemisphere (van de Hulst, 1980):

$$
f=\frac{1}{2} \int_{0}^{1} \phi(\cos \alpha) d(\cos \alpha)
$$

In Figure 2 the values of $f$ and $g$ computed from Mie theory for a wide range of spherical particles of silicate, graphite and dirty ice composition are compared with the $f-g$ relation for the HG phase function. Again, the HG phase function is an acceptable representation for all sizes except those with significant back-scattering peaks. This is not serious, because experimental evidence suggests that irregular, 


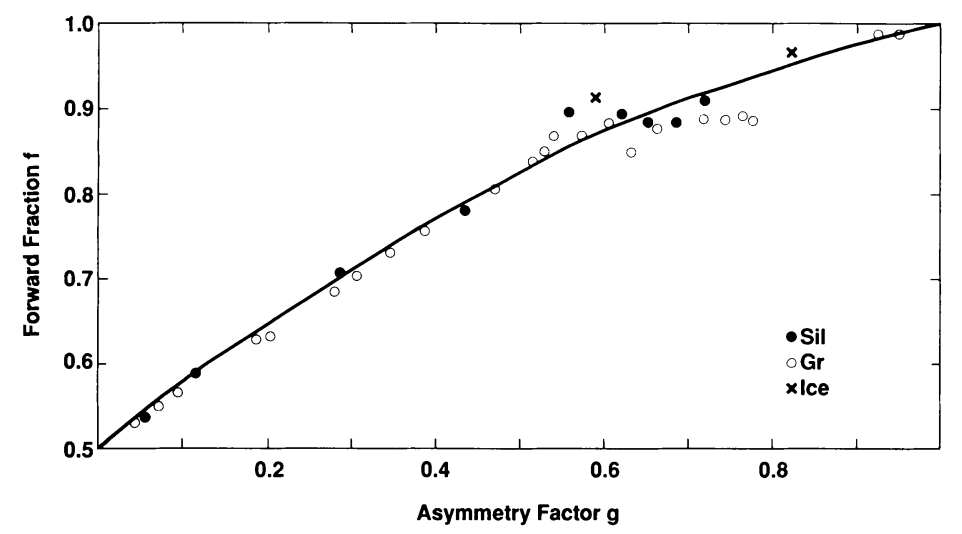

Fig. 2. The relationship between the fraction of light scattered into the forward hemisphere and the phase function asymmetry parameter $g$ for the Henyey-Greenstein phase function (solid curve) and for a series of Mie theory calculations for a range of sizes of silicate (Sil), graphite (Gr) and dirty ice (Ice) grains.

randomly oriented grains do not show the back-scattering peak predicted by Mie theory for spherical particles (Holland and Draper, 1967) or show it in significantly weakened form (Chylek, Grams, and Pinnick, 1976). Also, calculations for randomly oriented spheroidal particles by Asano and Sato (1980) show an insignificant back-scattering peak compared to predictions for area-equivalent spheres. We may conclude that, for a size distribution of realistic interstellar grains, the HG phase function describes the redistribution of the scattered light quite accurately.

\section{DERIVATION OF SCATTERING PROPERTIES}

\subsection{GENERAL}

The determination of the albedo and of the phase function asymmetry of interstellar dust usually involves much greater uncertainties than, for example, the derivation of an extinction curve. Not only are the observations often difficult, requiring intensity measurements in extended, diffuse sources with brightness levels comparable to the brightness of the night sky itself, but the actual derivation of scattering properties requires a radiative transfer model, which assumes the knowledge of the geometry of the scatterers and of the illuminating radiation field. The analysis is further complicated by multiple-scattering, which tends to become important in brighter sources with optical depths of order 0.3 or more.

Observations of a scattering system from a single aspect usually do not result in a unique solution but rather in a family of solutions with a range of $a$ and $g$ values. Observations of several scattering systems, or of several aspects of the same system, must be combined to narrow the range of final solutions. For example, the diffuse galactic light intensity at intermediate galactic latitudes $17^{\circ} \leq|b| \leq 20^{\circ}$ depends almost entirely on the dust albedo (Witt and Lillie, 1973), while the surface 


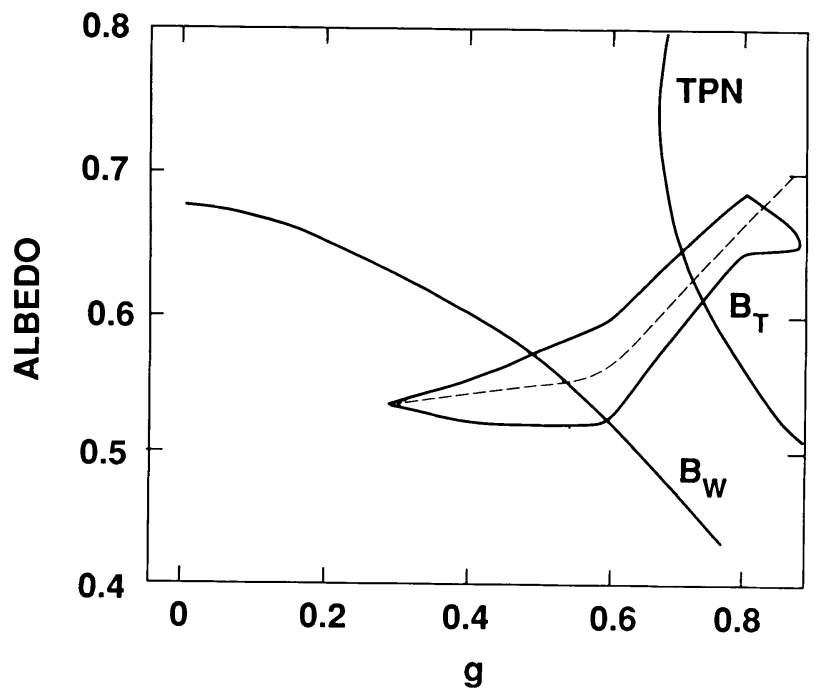

Fig. 3. Scattering properties of interstellar dust derived from observations in the $B$-band of the diffuse galactic light (DGL) by Witt (1968) $\left(\mathrm{B}_{\mathrm{W}}\right)$ and Toller (1981) $\left(\mathrm{B}_{\mathrm{T}}\right.$; dashed curve with solid boundary) and of the Thumbprint nebula (TPN) by FitzGerald, Stephens, and Witt (1976).

brightness profiles of globules depend mainly on the phase function asymmetry (FitzGerald, Stephens, and Witt, 1976). Similarly, the different solution families in the $a-g$ plane for the surface brightness of dark nebulae at low and high galactic latitudes were used by Mattila $(1970 a, 1970 b)$ to arrive at a unique set of scattering parameters.

\subsection{THE DIFFUSE GALACTIC LIGHT}

The scattering of the general interstellar radiation field by the dust contained in diffuse clouds, which gives rise to the diffuse galactic light (DGL), has received recent attention through the work of Toller (1981). By using all-sky observations made with the interplanetary probe Pioneer 10 at heliocentric distances greater than $3 \mathrm{AU}$, Toller was able to avoid major difficulties of ground-based measurements of the DGL encountered in earlier studies by Witt (1968) and Roach et al. (1972), such as zodiacal light, variable airglow, and atmospheric scattering. Toller's results from his $B\left(\lambda_{\text {eff }} \simeq 4400 \AA\right)$ data are shown in Figure 3, together with the solution derived from Witt's (1968) data by van de Hulst and de Jong (1969), and the result found by FitzGerald, Stephens and Witt (1976) for the Thumbprint nebula, a dense globule illuminated by the interstellar radiation field. All three results are consistent with Toller's conclusion of $a=0.61 \pm 0.07$ and $g=0.66 \pm 0.22$, although the TPN curve favors $g$-values near the upper limit of the range. Scattering properties derived from the DGL have the obvious advantage that they represent a "galactic average", against which exceptional scattering characteristics found in individual clouds may be compared. 


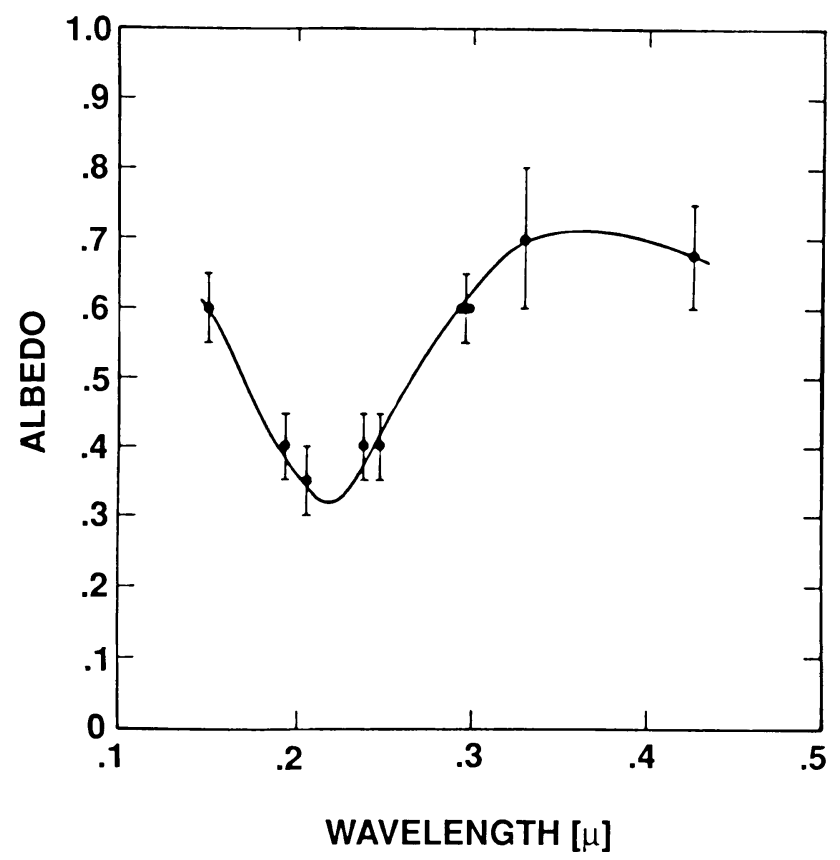

Fig. 4. The wavelength dependence of the albedo of galactic dust as derived from OAO-2 observations by Lillie and Witt (1976).

In the ultraviolet, DGL studies at low galactic latitudes by Lillie and Witt (1976) with the OAO-2 satellite, and by Morgan, Nandy and Thompson (1978) with the TD-1 satellite, have defined the wavelength dependence of the albedo reasonably well. The albedo minimum seen near $2200 \AA$ in the Lillie and Witt albedo curve, shown in Figure 4, is consistent with a pure absorption nature of the $2175 \AA$ band seen in interstellar extinction.

\subsection{THE FAR-UV BACKGROUND AT HIGH GALACTIC LATITUDES}

DGL data in the UV measured at low galactic latitudes do not constrain the phase function asymmetry particularly well, mainly because the dust near the galactic plane is illuminated from all directions. Dust situated at high $z$-distances in the Galaxy, on the other hand, is illuminated predominantly by OB stars near the central plane, and the intensity of the back-scattered light should be a sensitive function of the phase function asymmetry of the scattering grains.

Despite extensive efforts, reported measurements of the diffuse background intensity at high galactic latitudes are in considerable disagreement. Uncertainties in the level of various spurious signals, such as residual airglow and light from stars in the field of view, which must be subtracted from the measurements, are very likely causes for the lack of agreement. 
The conflicting results can be grouped approximately into two classes. One maintains that the high-latitude UV background has intensities of order 300 photons $\mathrm{cm}^{-2}-\mathrm{s}^{-1}-\AA^{-1}-\mathrm{sr}^{-1}$ and is not correlated with $\mathrm{HI}$ column density (Henry et al., 1978, 1980; Anderson et al., 1979, 1982; Tennyson et al., 1988; Weller, 1983), while the other suggests a highly patchy background with intensities ranging from 300 to 6000 photons $\mathrm{cm}^{-2}-\mathrm{s}^{-1}-\AA^{-1}-\mathrm{sr}^{-1}$ with positive correlations with measured $H \mathrm{I}$ column densities (Paresce et al., 1979; Paresce, McKee and Bowyer, 1980; Joubert et al., 1983; Jakobsen et al., 1984; Hurwitz, Martin and Bowyer, 1986).

In the analysis of the diffuse UV background radiation (e.g. Joubert et al., 1983; Jakobsen, de Vries and Paresce, 1987; Tennyson et al., 1988) frequent use is made of an approximate formula for the scattered light intensity from a cloud located above the Galactic disk, originally derived by Jura (1979). This formula has only a narrow range of validity, and its inappropriate use is adding to the confusion already present in this field. A more fully developed radiative transfer treatment of the high-latitude background radiation in the UV is very much needed.

\section{REFLECTION NEBULAE}

\subsection{SCATTERING IN CLASSICAL REFLECTION NEBULAE}

I consider reflection nebulae almost ideal laboratories for the study of the interaction of dust and radiation in interstellar space. They are observable from the Lyman limit to the sub-mm wavelength range; many nebulae can be found with a single, well-known source of illumination; the surface brightness in the visible and UV is high compared to that of the DGL; their geometry, again in contrast to the DGL, can be considered wavelength-independent, making them ideally suited for studies of the variation of scattering properties with wavelengths; and finally, when studying the brightest reflection nebulae available, their geometry must be close to the ideal geometry for maximizing the surface brightness, i.e. a star embedded in a uniform nebula by an optical depth of order unity. A disadvantage, but also an opportunity, arises from the fact that with each nebula the dust properties of only one cloud are being studied.

In order to fully exploit the potential of reflection nebulae, one needs to carry out accurate surface photometry over the entire nebular extent at widely-spaced wavelengths. In the UV, it has only been possible so far to adequately map the Merope nebula (Andriesse, Piersma and Witt, 1977; Witt, Bohlin and Stecher, 1986) and to obtain some crude estimates of the brightness distribution in NGC 7023 (Witt et al., 1982) by combining results from several satellite experiments. Once UV surface brightness maps of reflection nebulae are routinely obtained, considerable insight into UV scattering properties will be gained.

Space limits us here to review only selected recent results. Figure 5 shows a UV spectrum of the Merope nebula after it has been divided by the energy distribution of the illuminating star. For comparison, three model curves are shown based on different extinction laws but with the assumption that the dust albedo and the phase function asymmetry remain constant with wavelength. Because the reflection nebula spectrum traces the wavelength dependence of the scattering amplitude, the absence of a $2175 \AA$ bump in the observed data is strong evidence for the absorption nature of that feature. The extremely blue far-UV portion of the spectrum is 


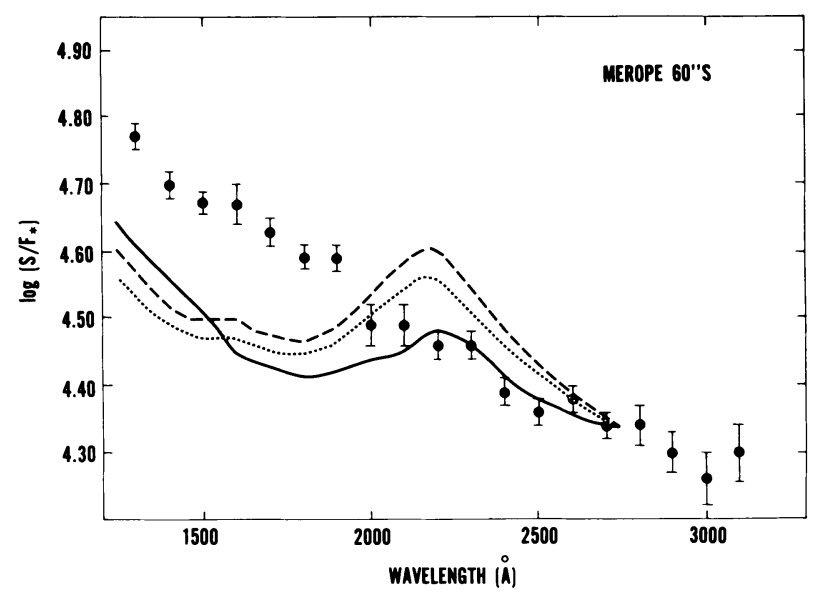

Fig. 5. The scattering amplitude of dust in the Merope nebula (filled circles) as observed by IUE at $60^{\prime \prime}$ south of Merope. Three model curves represent expectations for different geometries and extinction curves with wavelength-independent values for the albedo and the asymmetry parameter.

believed to point toward a less forward-scattering phase function at the shortest observed wavelengths, characteristic of particles small compared to the wavelength (Witt, Bohlin and Stecher, 1986a; Witt, 1985). A useful compilation of reflection nebula spectra in the UV, which demonstrate the extreme range of nebular colors, was published by Cardelli and Böhm (1984).

Two reflection nebulae, CED 201 and IC 435, have recently been shown to exhibit enhanced scattering in the long-wavelength wing of the $2175 \AA$ feature (Witt, Bohlin and Stecher, 1986b; Witt et al., 1987). In Figure 6, the ratios of the spectra of CED 201 and IC 435 and those of their respective illuminating stars are shown, with the vertical arrow indicating the central wavelength of the $2175 \AA$ feature. Scattering in the long-wavelength wing of a resonance is characteristic of grains of larger radius than normally invoked to explain the $2175 \AA$ feature in absorption. As an example, the wavelength dependences of albedo and phase function asymmetry of a spherical graphite grain of radius $0.05 \mu \mathrm{m}$ are shown in Figure 7 . Such a grain reaches an albedo maximum of 0.6 near $2600 \AA$ and becomes strongly forward-scattering at still shorter wavelengths, which, in an appropriate geometry, can produce an effect similar to the observed one. Graphite grains normally used in models to produce the $2175 \AA$ feature have radii of $0.02 \mu \mathrm{m}$ or less, and an albedo of less than 0.16. This example does not prove that graphite grains are the agent responsible for the $2175 \AA$ feature, but it strongly suggests that the $2175 \AA$ band is the result of a solid state effect involving extinction by grains, not absorption by molecules. 


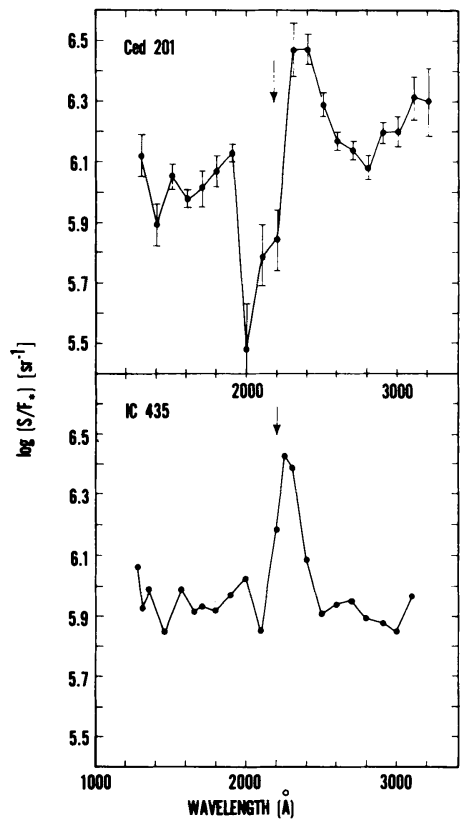

Fig. 6. Evidence for scattering in the $2175 \AA$ feature, as observed with IUE in the two reflection nebulae Ced 201 and IC 435 . The arrow indicates the $2175 \AA$ position.

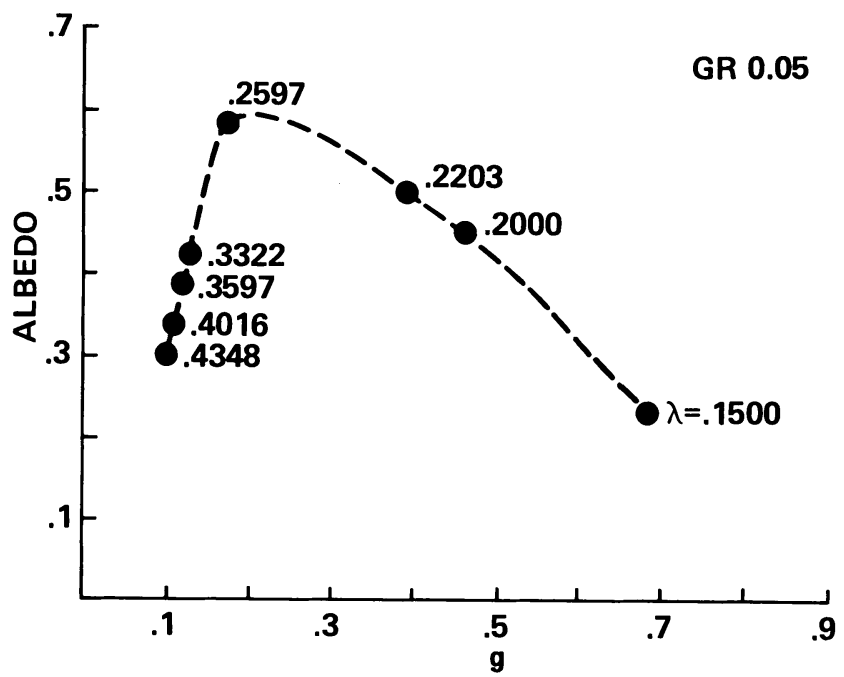

Fig. 7. The computed wavelength dependence of the albedo and the asymmetry parameter $g$ for a spherical graphite grain of $0.05 \mu \mathrm{m}$ radius. The wavelengths are in units of $\mu \mathrm{m}$. 


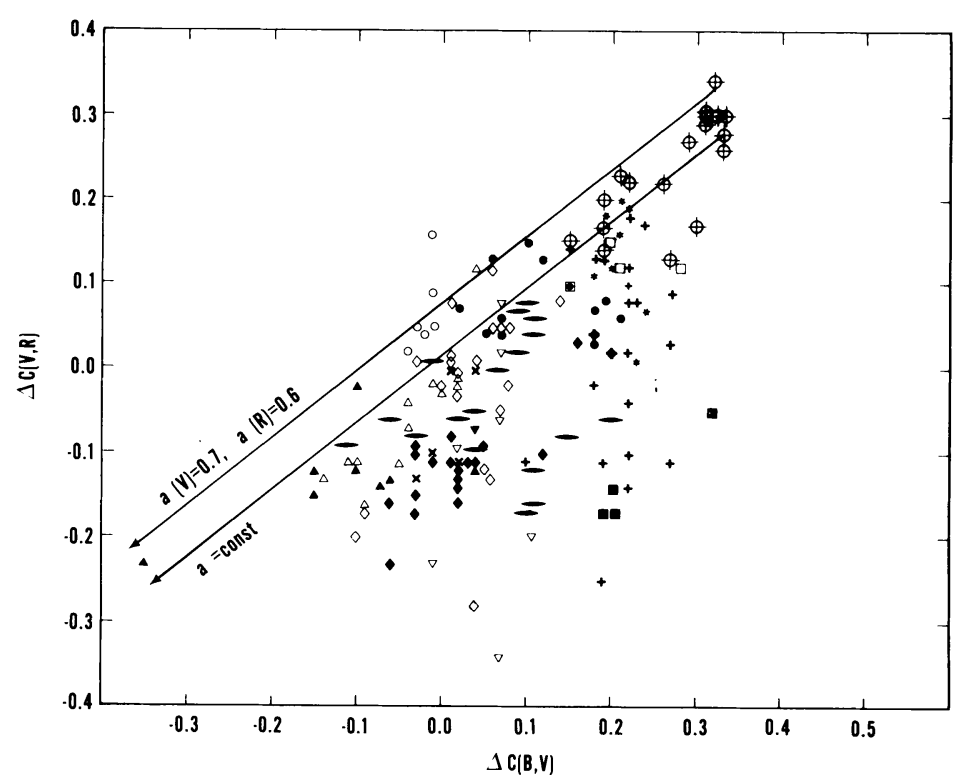

Fig. 8. Observed color differences $\Delta(V, R)$ are plotted against corresponding color differences $\Delta(B, V)$ for 12 reflection nebulae. The solid lines indicate the expected relationship for either constant albedo or an albedo decreasing with increasing wavelength.

\subsection{DUST LUMINESCENCE: EXTENDED RED EMISSION}

It has now been established by several observational techniques that interstellar dust, under not yet fully understood conditions, will exhibit luminescence in the $5500 \AA-9000 \AA$ wavelength range. This luminescence, termed "extended red emission" (ERE) takes the form of a broad band, peaking near $6700 \AA$ with about $1200 \AA$ FWHM. The ERE can contribute as much as $35 \%$ to the surface brightness of a reflection nebula in the $R$ band. This causes affected nebular regions to appear substantially redder in the $V-R$ color than expected on the basis of their $B-V$ color, as is demonstrated in Figure 8. Here the color difference $\Delta C(V, R)=\log \left(S / F_{*}\right)_{V}-\log \left(S / F_{*}\right)_{R}$ is plotted against the corresponding color difference $\Delta C(B, V)$ for multiple observations in 12 nebulae. A similar effect is seen in the $I\left(\lambda_{\text {eff }}=8800 \AA\right)$ photometric band (Witt and Schild, 1986; Witt, Schild and Kraiman, 1984).

The association of the red color with a luminescence band has been confirmed by nebular spectroscopy (Witt and Schild, 1988; Witt, Boroson, and Schild, 1988). The reddest regions show the strongest ERE band. In Figure 9 this band is illustrated for NGC 7023. Shown here is the ratio of the nebular flux (from a field approximately $60^{\prime \prime} \mathrm{NNW}$ of HD 200775) and the stellar flux, expressed in magnitudes. This band is probably the same as first seen in the Red Rectangle nebula by Schmidt, Cohen and Margon (1980), except that its relative intensity is much lower in nebulae other than the Red Rectangle. The band was found to peak near $6400 \AA$ in the 


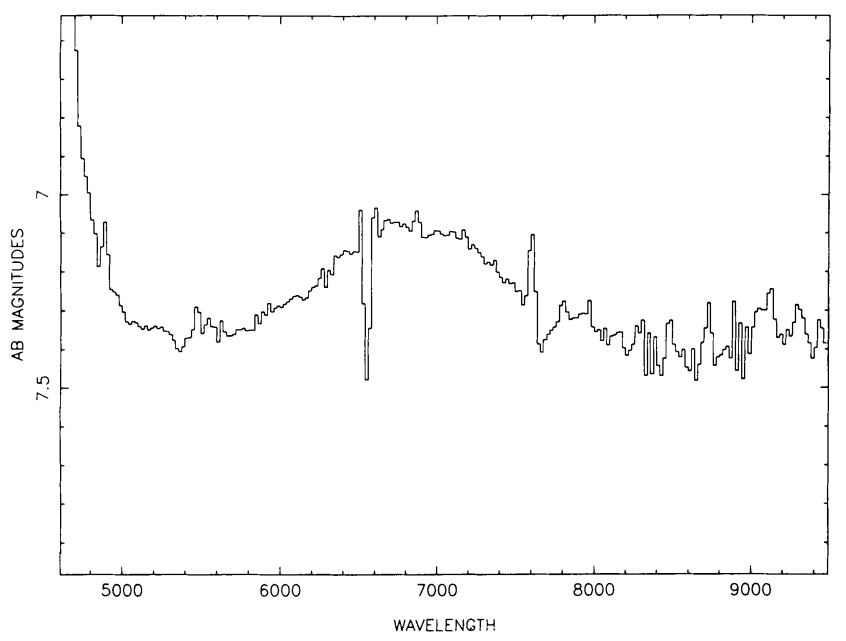

Fig. 9. The extended red emission (ERE) feature peaking near $6800 \AA$, as observed $60^{\prime \prime} N N W$ of HD 200775 in NGC 7023.

Red Rectangle, while in reflection nebulae studied since, the ERE band reaches its maximum at wavelengths from $6600 \AA$ to $7100 \AA$. This behavior, including the observed wavelength shift of the emission maximum with distance from the illuminating star seen in some objects, is important for the identification of the ERE emitter.

Careful analysis of color variations in NGC 2023 and NGC 7023 has led to the identification of the likely excitation source for the ERE: mid-UV photons from the illuminating star (Witt and Schild, 1985). This is consistent with the observed fact that nebulae illuminated by early A stars still exhibit ERE. Possible evidence for the presence of ERE in an isolated dark cloud, illuminated by the diffuse interstellar radiation field, has been discussed by Chlewicki and Laureijs (1987).

In the best-studied reflection nebula, NGC 2023, it has been shown that the spatial distribution of ERE is positively correlated with the distribution of $\mathrm{H}_{2}$ photodissociation regions and regions bright in $3.3 \mu \mathrm{m}$ emission. (Witt and Schild, 1988; Gatley et al., 1987). This has been further confirmed by very recent results on two nebulae, IC 59 and IC 63, associated with $\gamma$ Cas (Witt, Stecher, Boroson and Bohlin, 1989), where $\mathrm{H}_{2}$-dissociation luminescence and ERE have been found together in IC 63, while both types of emission are absent in IC 59.

An identification of the ERE with photo-luminescence by hydrogenated amorphous carbon has been suggested (Duley, 1985, 1988; Witt and Schild, 1988), and supported by excellent agreement with the laboratory data of Watanabe, Hasegawa and Kurata (1982). The ERE band is matched in both wavelength position and width, and the change of peak wavelength with environment is explained as resulting from changes in the degree of hydrogenation, which affects the bandgap of the material. An alternative explanation for the ERE has been advanced by d'Hendecourt et 
al. (1986) in terms of fluorescence by free polycyclic aromatic hydrocarbon (PAH) molecules, however without supporting laboratory evidence.

\subsection{THE FAR-INFRARED EMISSION EFFICIENCY}

The ratio of the far-infrared dust absorption efficiency to the extinction efficiency in the UV and visible provides important information on the structure of interstellar grain material. As discussed by Seki and Yamamoto (1980), crystalline dielectrics as well as metals have far-infrared emission or absorption efficiencies varying as (radius $/ \lambda^{2}$ ), whereas amorphous solids exhibit a $\lambda^{-1}$ wavelength dependence in their far-infrared emission efficiency. As an example, laboratory data for amorphous carbon grains by Koike, Hasegawa and Manabe (1980) agree well with the expected $\lambda^{-1}$ dependence, while graphite properties computed with Sato's (1968) indices of refraction agree with the expected $\lambda^{-2}$ dependence.

Reflection nebulae and other systems illuminated by sources of known flux have been used for empirical determinations of the ratio of the far-IR to UV/visible absorption efficiency ratios, $Q_{a b s}(V) / Q_{a b s}(I R)$. Werner et al. (1978) found $Q_{a b s}(V)$ $/ Q_{a b s}(I R) \simeq 1000$ for dust in the 30 Dor region, while Becklin et al. (1976) estimate $Q_{a b s}(V) / Q_{a b s}(I R) \simeq 100$ for the Orion ionization front. The globule B335 yielded a value $Q_{e x t}(V) / Q_{a b s}(I R)>700$ in the investigation by Keene (1981), and the study of NGC 7023 by Whitcomb et al. (1981) produced $Q_{e x t}(V) / Q_{a b s}(I R) \simeq 2000$ 5000. While all these results are subject to considerable uncertainty, they generally are within the range expected for a $\lambda^{-1}$ dependence in $Q_{a b s}$. The $\lambda^{-2}$ dependence for crystalline grains would predict $Q_{a b s}(V) / Q_{a b s}(I R) \simeq 7 \times 10^{5}$. These results, therefore, support the idea that interstellar grains have an amorphous nature.

\subsection{THE SURFACE BRIGHTNESS PROFILES OF GLOBULES}

Isolated dark nebulae and Bok globules, when observed against the darker sky at higher galactic latitudes, exhibit surface brightness profiles characterized by dark cores surrounded by brighter rims. This phenomenon results when the galactic interstellar radiation field is scattered by non-homogeneous, centrally condensed clouds containing dust with a strongly forward directed phase function (Witt and Stephens, 1974). Indeed, the dark cores of globules are the least model-dependent evidence for the asymmetry of the scattering phase function, as was first recognized by Struve (1937).

The study of such globules is therefore a means of determining dust scattering properties in quiescent, dense interstellar clouds, while simultaneously yielding information on density distributions and cloud masses. FitzGerald, Stephens and Witt (1976) carried out an analysis of the Thumbprint nebula in the $B$ and $V$ bandpasses with the result that $a$ and $g$ both have probable values of $0.7 \pm 0.1$. The density in the cloud drops off as $r^{-2}$ from a constant-density core, with a cloud mass of $9 \mathbf{M}_{\odot}$.

Recently, Oliveri, Schild and Witt (1987) extended such studies to the near-IR with a globule first described by Dickman and Clemens (1983). They found albedo values of $0.6 \pm 0.1$ for the $R$ and $I$ bands and a phase function asymmetry of $0.60 \leq g \leq 0.8$. The high albedo and strong asymmetry at these longer wavelengths 
suggests that fairly large particles with diameters of about $1.0 \mu \mathrm{m}$ must be abundant enough to dominate the scattering in such clouds.

ACKNOWLEDGEMENTS. This work has been supported by NSF Grant AST 8418183 and by NASA Grant NAG5-1065, both to The University of Toledo.

\section{REFERENCES}

Anderson, R. C., Henry, R. C., Brune, W. H., Feldman, P. D., and Fastie, W. G. 1979, Ap. J., $284,415$.

Anderson, R. C., Henry, R. C., and Fastie, W. G. 1982, Ap. J., 259, 573.

Andriesse, C. D., Piersma, Th. R., and Witt, A. N. 1977, Astr. Ap., 54, 841.

Becklin, E. E., Beckwith, S., Gatley, I., Matthews, K., Neugebauer, G., Sarasin, C., and Werner, M. W. 1976, Ap. J., 207, 770.

Cardelli, J. A., and Böhm, K. -H. 1984, Ap. J., $285,613$.

Chlewicki, G. and Laureijs, R. J. 1987, in Polycyclic Aromatic Hydrocarbons and Astrophysics, eds. A. Léger, L. d'Hendecourt, and N. Boccara, (Dordrecht: Reidel), p. 335.

Chylek, P., Grams, G. W., and Pinnick, R. G. 1976, Science, 198, 480.

Dickman, R. L., and Clemens, D. P. 1983, Ap. J., $271,143$.

Duley, W. W. 1985, M. N. R. A. S., 215, 259.

. $1988, \dot{M} . N . R . A . S$., in press.

Elvey, C. T., and Roach, F. E. 1937, Ap. J., 85, 213.

FitzGerald, M. P., Stephens, T. C., and Witt, A. N. 1976, Ap. J., 208, 709.

Gatley, I. et al. 1987, Ap. J. (Letters), 818, L73.

Henry, R. C., Anderson, R. C., Feldman, P. D., and Fastie, W. G. 1978, Ap. J., 222, 902.

Henry, R. C., Anderson, R. C., and Fastie, W. G. 1980, Ap. J., $239,859$.

Henyey, L. G., and Greenstein, J. L. 1941, Ap. J., 98, 70.

Holland, A. C., and Draper, J. S. 1967, Appl. Opt., 6, 511.

Hubble, E. 1922, Ap. J., 56, 400.

Hurwitz, M., Martin, C., and Bowyer, S. 1986, Adv. Space Res., 6, 2.

Jakobsen, P., Bowyer, S., Kimble, R., Jelinsky, P., Grewing, M., Krämer, G., and Wulf-Mathis, C. 1984, Astr. Ap., 139, 481.

Jakobsen, P., de Vries, J. S., and Paresce, F. 1987, Astr. Ap., $183,335$.

Joubert, M., Masnou, J. L., Lequeux, J., Deharveng, J. M., and Cruvellier, P. 1983, Astr. Ap., 128, 114 .

Jura, M. 1979, Ap. J., 227, 798.

Keene, J. 1981, Ap. J., 245, 115.

Koike, C., Hasegawa, H., and Manabe, A. 1980, Ap. Space Sci., 67, 495.

Lillie, C. F. and Witt, A. N. 1976, Ap. J., 208, 64.

Mattila, K. 1970a, Astr. Ap., 8, 273.

. 19706, Astr. Ap., $9,53$.

Morgan, D. H., Nandy, K., and Thompson, G. I. 1978, M. N. R. A. S., 185, 371.

Oliveri, M. V., Schild, R. E., and Witt, A. N. 1987, Bull. A. A. S., 19, 1055.

Paresce, F., Margon, B., Bowyer, S., and Lampton, M. 1979, Ap. J., $230,304$.

Paresce, F., McKee, C., and Bowyer, S. 1980, Ap. J., 240, 387 .

Roach, F. E., Smith, L. L., Pfleiderer, J., Batishko, C., and Batishko, K. 1972, Ap. J., $178,343$.

Sato, Y. 1968, J. Phys. Soc. Japan, 24, 489.

Schmidt, G. D., Cohen, M., and Margon, B. 1980, Ap. J. (Letters), 289, L133.

Seki, J. and Yamamoto, T. 1980, Ap. Space Sci., $72,79$.

Slipher, V. M. 1912, Lowell Obs. Bull., 11, No. 55, 26.

Struve, O. 1937, Ap. J., 85, 194.

Tennyson, P. D., Henry, R. C., Feldman, P. D. and Hartig, G. F. 1988, Ap. J., 330, 435.

Toller, G. N. 1981, A Study of Galactic Light, Extragalactic Light, and Galactic Structure using Pioneer 10 Observations of Back-ground Starlight., Ph.D. Thesis, State University of New York at Stony Brook.

van de Hulst, H. C. 1980, Multiple Light Scattering, Tables, Formulas, and Applications, (New York: Academic Press), vol. 2, p. 305. 
Watanabe, I., Hasegawa, S., and Kurata, Y. 1982, Japan J. Appl. Phys., 21, 856.

Weller, C. S. 1983, Ap. J., 268, 899.

Werner, M. W., Becklin, E. E., Gatley, I., Ellis, M., Hyland, A. R., Robinson, G., and Thomas, J. A. $1978, M$. N. R. A. S., 184,365 .

Whitcomb, S. E., Gatley, I., Hildebrand, R. H., Keene, J., Sellgren, K., and Werner, M. W. 1981, Ap. J., 246, 416.

Witt, A. N. 1968, Ap. J., 152, 59.

1985, Ap. J., 294, 216.

Witt, A. N., Bohlin, R. C., and Stecher, T. P. 1986a, Ap. J., 302, 421.

1986 b. Ap. J. (Letters), 805, L23.

Witt, A. N., Bohlin, R. C., Stecher, T. P., and Graff, S. M. 1987, Ap. J., s21, 912.

Witt, A. N., Boroson, T. A., and Schild, R. E. 1988, in Interstellar Matter, Proceedings of the Second Haystack Observatory Meeting, eds. J. Moran and P. Ho, (New York: Gordon and Breach), p. 241.

Witt, A. N. and Lillie, C. F. 1973, Astr. Ap., 25, 397.

Witt, A. N. and Schild, R. E. 1985, Ap. J., 294, 225. 1986, Ap. J. Suppl., 62, 839. 1988, Ap. J., $325,837$.

Witt, A. N., Schild, R. E., and Kraiman, J. B. 1984, Ap. J., 281, 708.

Witt, A. N. and Stephens, T. C. 1974, A. J., 79, 948.

Witt, A. N., Walker, G. A. H., Bohlin, R. C., and Stecher, T. P. 1982, Ap. J., $261,492$. 